\title{
BINARY FORMS, HYPERGEOMETRIC FUNCTIONS AND THE SCHWARZ-CHRISTOFFEL MAPPING FORMULA
}

\author{
MICHAEL A. BEAN
}

\begin{abstract}
In a previous paper, it was shown that if $F$ is a binary form with complex coefficients having degree $n \geq 3$ and discriminant $D_{F} \neq 0$, and if $A_{F}$ is the area of the region $|F(x, y)| \leq 1$ in the real affine plane, then $\left|D_{F}\right|^{1 / n(n-1)} A_{F} \leq 3 B\left(\frac{1}{3}, \frac{1}{3}\right)$, where $B\left(\frac{1}{3}, \frac{1}{3}\right)$ denotes the Beta function with arguments of $1 / 3$. This inequality was derived by demonstrating that the sequence $\left\{M_{n}\right\}$ defined by $M_{n}=\max \left|D_{F}\right|^{1 / n(n-1)} A_{F}$, where the maximum is taken over all forms of degree $n$ with $D_{F} \neq 0$, is decreasing, and then by showing that $M_{3}=3 B\left(\frac{1}{3}, \frac{1}{3}\right)$. The resulting estimate, $A_{F} \leq 3 B\left(\frac{1}{3}, \frac{1}{3}\right)$ for such forms with integer coefficients, has had significant consequences for the enumeration of solutions of Thue inequalities.

This paper examines the related problem of determining precise values for the sequence $\left\{M_{n}\right\}$. By appealing to the theory of hypergeometric functions, it is shown that $M_{4}=2^{7 / 6} B\left(\frac{1}{4}, \frac{1}{2}\right)$ and that $M_{4}$ is attained for the form $X Y\left(X^{2}-Y^{2}\right)$. It is also shown that there is a correspondence, arising from the Schwarz-Christoifel mapping formula, between a particular collection of binary forms and the set of equiangular polygons, with the property that $A_{F}$ is the perimeter of the polygon corresponding to $F$. Based on this correspondence and a representation theorem for $\left|D_{F}\right|^{1 / n(n-1)} A_{F}$, it is conjectured that $M_{n}=$ $D_{F_{n}^{*}}^{1 / n(n-1)} A_{F_{n}^{*}}$, where $F_{n}^{*}(X, Y)=\prod_{k=1}^{n}\left(X \sin \left(\frac{k \pi}{n}\right)-Y \cos \left(\frac{k \pi}{n}\right)\right)$, and that the limiting value of the sequence $\left\{M_{n}\right\}$ is $2 \pi$.
\end{abstract}

\section{INTRODUCTION}

Let $F(X, Y)=a_{0} X^{n}+a_{1} X^{n-1} Y+\cdots+a_{n} Y^{n}$ be a binary form with complex coefficients and let $A_{F}$ denote the area of the region $|F(x, y)| \leq 1$ in the real affine plane. Let $D_{F}$ denote the discriminant of $F$. If $F$ has the factorization $\prod_{i=1}^{n}\left(\alpha_{i} X-\beta_{i} Y\right)$ with $\alpha_{i}, \beta_{i} \in \mathbb{C}$ (every binary form has such a factorization), then $D_{F}=\prod_{i<j}\left(\alpha_{i} \beta_{j}-\alpha_{j} \beta_{i}\right)^{2}$.

In a previous paper [2], we derived an isoperimetric inequality for the quantity $A_{F}$. To be specific, we showed that if $F$ is a binary form with complex coefficients having degree $n \geq 3$ and discriminant $D_{F} \neq 0$, then

$$
\left|D_{F}\right|^{1 / n(n-1)} A_{F} \leq 3 B\left(\frac{1}{3}, \frac{1}{3}\right)
$$

Received by the editors June 23, 1994 and, in revised form, December 21, 1994.

1991 Mathematics Subject Classification. Primary 11D75, 11J25; Secondary 26D20, 30C20, $33 \mathrm{C} 05,51 \mathrm{M} 25$. 
where $B\left(\frac{1}{3}, \frac{1}{3}\right)$ denotes the Beta function with arguments of $1 / 3$. Moreover, we showed that the bound $3 B\left(\frac{1}{3}, \frac{1}{3}\right)$, which has an approximate numerical value of 15.8997 , is attained precisely when $F$ is a cubic form which, up to multiplication by a complex number, is equivalent under $G L_{2}(\mathbb{R})$ (in the sense defined below) to the form $X Y(X-Y)$. by

We derived inequality (1) by demonstrating that the sequence $\left\{M_{n}\right\}$ defined

$$
M_{n}=\max \left|D_{F}\right|^{1 / n(n-1)} A_{F},
$$

where the maximum is taken over all forms of degree $n$ with $D_{F} \neq 0$, is decreasing, and then by showing that $M_{3}=3 B\left(\frac{1}{3}, \frac{1}{3}\right)$. In this paper, we will examine more closely the nature of the sequence $\left\{M_{n}\right\}$. In particular, we will address the following three questions which arise naturally from the preceding results:

- What is the value of $M_{n}$ for $n>3$ ?

- Are there canonical classes of forms $F_{n}$ for which

$$
M_{n}=\left|D_{F_{n}}\right|^{1 / n(n-1)} A_{F_{n}}
$$

for $n>3$ ?

- What is the limiting behavior of the sequence $\left\{M_{n}\right\}$ ?

While we are unable to give complete answers to these questions, we will give some striking heuristics which suggest that each $M_{n}$ is attained by the form of degree $n$ whose graph has the most symmetries and that the limiting value of the sequence $\left\{M_{n}\right\}$ is $2 \pi$. One of the heuristics, which is interesting in its own right, involves the use of the Schwarz-Christoffel mapping formula to give a correspondence between the areas defined by certain binary forms and the perimeters of equiangular polygons.

As mentioned in the previous work [2], the estimate for $A_{F}$ given in (1) has significant implications in the enumeration of solutions of Thue inequalities. A Thue inequality is a Diophantine inequality of the type $|F(x, y)| \leq h$ where $F$ is a binary form with integer coefficients which is irreducible and has degree $n \geq 3$, and $h$ is a non-zero integer. In a seminal paper of 1909, Thue [17] showed that for such a form $F$, the equation $F(x, y)=h$ (hence the inequality $|F(x, y)| \leq h)$ has only a finite number of solutions in integers $x$ and $y$. In 1934, Mahler [11] showed that the number, $N_{F}(h)$, of solutions of the Thue inequality $|F(x, y)| \leq h$ and the area, $A_{F} h^{2 / n}$, of this region are related in the following way:

$$
\left|N_{F}(h)-A_{F} h^{2 / n}\right| \leq c h^{1 /(n-1)}
$$

where $c$ is a number (left unspecified by Mahler) which depends only on $F$. Estimates for $N_{F}(h)$ involving $A_{F} h^{2 / n}$ have also been given by Mueller and Schmidt [12].

Since the discriminant of a form with integer coefficients is an integer, inequality (1) implies that $A_{F}<16$ over the class of forms with integer coefficients having non-zero discriminant and degree at least three. In light of this result, one might expect the error in the approximation of $N_{F}(h)$ by $A_{F} h^{2 / n}$ to depend only on $h$. However, an examination of the forms

$$
P_{k}(X, Y)=X^{2 k}+(X-Y)^{2}(2 X-Y)^{2} \cdots(k X-Y)^{2}
$$




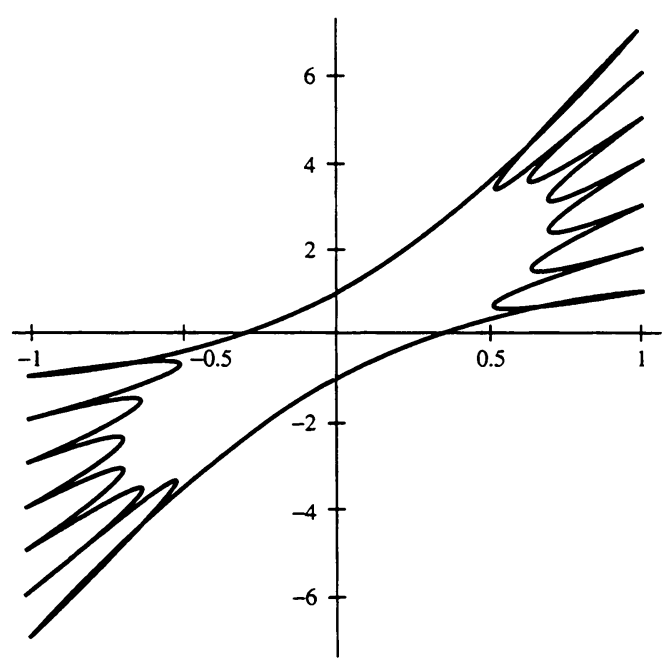

FIGURE 1. $\left|P_{7}(x, y)\right|=1$

reveals that this is not even the case when $h=1$. Indeed, the region $\left|P_{k}(x, y)\right|$ $\leq 1$ contains at least $2 k$ integer lattice points, namely

$$
\pm(1,1), \pm(1,2), \ldots, \pm(1, k)
$$

but nevertheless is bounded (since $P_{k}$ is positive definite) and has area less than 16. (See Figure 1 for the graph of $P_{7}$.) In a subsequent paper, we will consider the question of how well $A_{F} h^{2 / n}$ approximates $N_{F}(h)$. In the present paper, we confine our attention to the sequence $\left\{M_{n}\right\}$.

Let us begin by recalling some notation from [2]. Let $G L_{2}(\mathbb{R})$ denote the group of $2 \times 2$ real invertible matrices and let $S L_{2}(\mathbb{R})$ denote the subgroup of matrices with determinant \pm 1 . For each $T=\left(\begin{array}{c}a b \\ c d\end{array}\right) \in G L_{2}(\mathbb{R})$, let $F_{T}$ denote the binary form given by $F_{T}(X, Y)=F(a X+b Y, c X+d Y)$. Two forms $F$ and $G$ are said to be equivalent under $G L_{2}(\mathbb{R})$ if $G=F_{T}$ for some $T \in G L_{2}(\mathbb{R})$. Equivalence under $S L_{2}(\mathbb{R})$ is defined similarly. The quantity $\left|D_{F}\right|^{1 / n(n-1)} A_{F}$ is invariant with respect to $G L_{2}(\mathbb{R})$, that is, $\left|D_{F_{T}}\right|^{1 / n(n-1)} A_{F_{T}}=$ $\left|D_{F}\right|^{1 / n(n-1)} A_{F}$ for all $T \in G L_{2}(\mathbb{R})$. It is also invariant with respect to replacing $F$ by $k F$ for any complex number $k$, but is not invariant with respect to $G L_{2}(\mathbb{C})$ (see [2]). For any form $F$, the polynomial $F(x, 1)$ is said to have a root at infinity. denoted $\infty$, if $Y$ is a factor of $F(X, Y)$; similarly, the polynomial $F(1, y)$ has a root at infinity if $X$ is a factor of $F(X, Y)$. With this convention, the slopes of the asymptotes of the curve $|F(x, y)|=1$ are the real roots of the polynomial $F(1, y)$.

In Theorem 3 of [2], we showed that the quantity $\left|D_{F}\right|^{1 / n(n-1)} A_{F}$ is maximized over the class of forms of degree $n$ with complex coefficients and non-zero discriminant by a form with real coefficients for which the polynomial $F(x, 1)$ has $n$ distinct real roots. In fact, we showed that if $F$ is a form of degree $n$ for which $F(x, 1)$ has at least one non-real root, then 


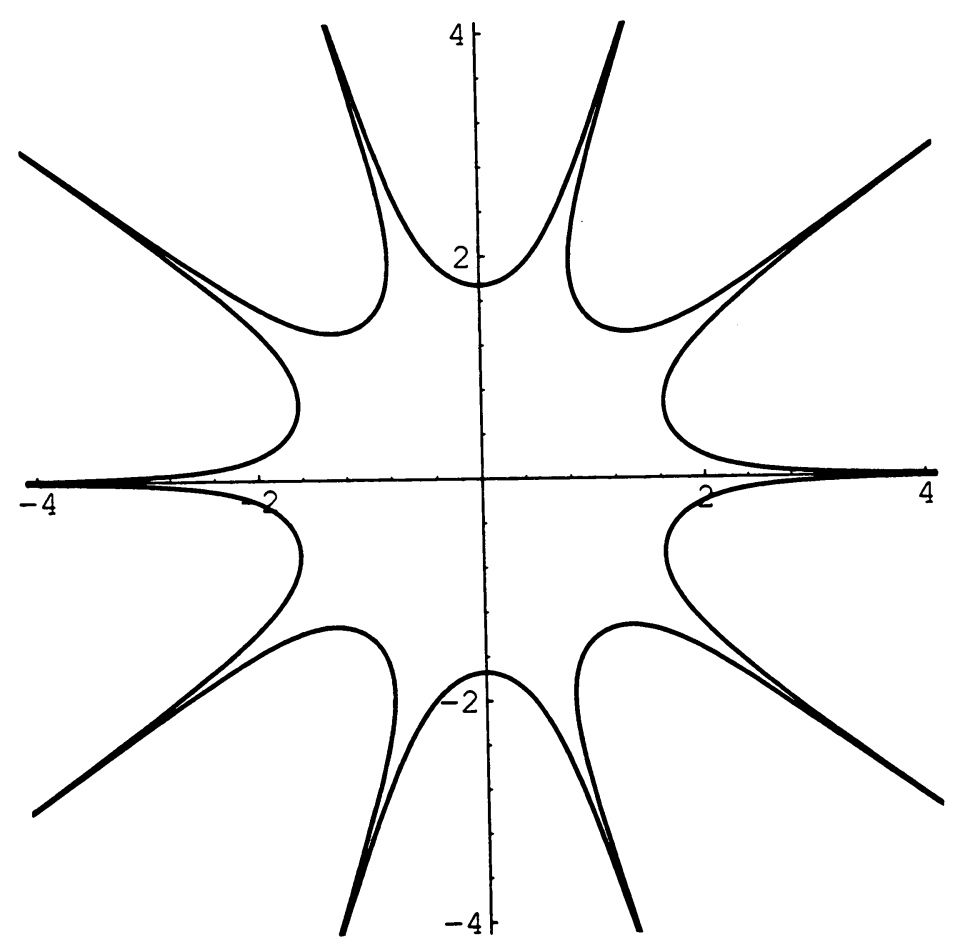

FIGURE 2. $\left|F_{5}^{*}(x, y)\right|=1$

$\left|D_{F}\right|^{1 / n(n-1)} A_{F}<M_{n}$. Hence, when considering questions about the sequence $\left\{M_{n}\right\}$, one may restrict attention to the forms $F$ with real coefficients for which $F(x, 1)$ has $n$ distinct real roots (counting any root at infinity). For simplicity, we will refer to such a form as one which has a complete factorization over $\mathbb{R}$. Notice that the discriminant of any such form is a positive real number.

In view of this theorem, it was a relatively straightforward matter to show that $M_{3}=3 B\left(\frac{1}{3}, \frac{1}{3}\right)$ since every cubic form with real coefficients and positive discriminant is equivalent under $G L_{2}(\mathbb{R})$ to the form $X Y(X-Y)$. However, evaluating $M_{n}$ for $n>3$ is not nearly so simple since, in general, the quantity $\left|D_{F}\right|^{1 / n(n-1)} A_{F}$ is not constant over the class of forms of degree $n$ which have a complete factorization over $\mathbb{R}$. Indeed, this quantity depends on $n-3$ independent quantities (as will soon become apparent).

A natural candidate to maximize $\left|D_{F}\right|^{1 / n(n-1)} A_{F}$ over the class of forms of degree $n$ is the form $F_{n}^{*}(X, Y)=\prod_{k=1}^{n}\left(X \sin \left(\frac{k \pi}{n}\right)-Y \cos \left(\frac{k \pi}{n}\right)\right)$. This form has the property that its graph $\left|F_{n}^{*}(x, y)\right|=1$ is invariant under every rotation which is an integer multiple of $\frac{\pi}{n}$ radians and is symmetric about each of the asymptotes $x \sin \left(\frac{k \pi}{n}\right)-y \cos \left(\frac{k \pi}{n}\right)=0, k=1,2, \ldots, n$. (See Figure 2 for the graph of $F_{5}^{*}$.)

In this paper, we will show that $M_{4}=D_{F_{4}^{*}}^{1 / 12} A_{F_{4}^{*}}=2^{7 / 6} B\left(\frac{1}{4}, \frac{1}{2}\right)$ by appealing to the theory of hypergeometric functions. We will also exhibit a correspondence between the collection of forms of degree $n$ having a complete factorization over $\mathbb{R}$ and the set of $n$-sided equiangular polygons, with the 
property that the perimeter of the polygon associated with $F$ is $A_{F}$. Under this correspondence, forms which are $G L_{2}(\mathbb{R})$-equivalent to $F_{n}^{*}$ are mapped to $n$-sided regular polygons. These results, together with a representation theorem for $D_{F}^{1 / n(n-1)} A_{F}$ over the restricted class, and numerical evidence, lead to the following conjectures.

Conjecture 1. The maximal value $M_{n}$ of the quantity $\left|D_{F}\right|^{1 / n(n-1)} A_{F}$ over the class of forms of degree $n$ with complex coefficients and non-zero discriminant is attained precisely when $F$ is a form which, up to multiplication by a complex number, is equivalent under $G L_{2}(\mathbb{R})$ to the form

$$
F_{n}^{*}(X, Y)=\prod_{k=1}^{n}\left(X \sin \left(\frac{k \pi}{n}\right)-Y \cos \left(\frac{k \pi}{n}\right)\right) .
$$

In particular,

$$
M_{n}=D_{F_{n}^{*}}^{1 / n(n-1)} A_{F_{n}^{*}} .
$$

Conjecture 2. The sequence of maximal values $\left\{M_{n}\right\}$ decreases monotonically to the value $2 \pi$.

Coincidentally, this conjectured limiting value is equal to the value $\left|D_{F}\right|^{1 / 2} A_{F}$ when $F$ is a quadratic form with real coefficients and negative discriminant.

\section{StATEMENT OF RESUlts}

Let $B(x, y)$ denote the Beta function of $x$ and $y$. The Beta function may be defined in terms of the Gamma function by the relation

$$
B(x, y)=\frac{\Gamma(x) \Gamma(y)}{\Gamma(x+y)}
$$

and has the integral representation

$$
B(x, y)=\int_{0}^{1} t^{x-1}(1-t)^{y-1} d t
$$

for $x>0$ and $y>0$ (see Abramowitz and Stegun [1]).

We will prove the following result regarding quartic forms.

Theorem 1. If $F$ is a binary quartic form with complex coefficients and non-zero discriminant, then

$$
\left|D_{F}\right|^{1 / 12} A_{F} \leq 2^{7 / 6} B\left(\frac{1}{4}, \frac{1}{2}\right) .
$$

This value is attained precisely when $F$ is a quartic form which, up to multiplication by a complex number, is equivalent under $G L_{2}(\mathbb{R})$ to the form $F_{4}^{*}(X, Y)=$ $\frac{1}{2} X Y\left(X^{2}-Y^{2}\right)$. In particular,

$$
M_{4}=D_{F_{4}^{*}}^{1 / 12} A_{F_{4}^{*}}=2^{7 / 6} B\left(\frac{1}{4}, \frac{1}{2}\right) .
$$

Since the sequence $\left\{M_{n}\right\}$ is decreasing, Theorem 1 provides an improved estimate for $\left|D_{F}\right|^{1 / n(n-1)} A_{F}$ when the degree of $F$ is greater than three. 
Corollary 1. If $F$ is a binary form of degree $n \geq 4$ with complex coefficients and non-zero discriminant, then

$$
\left|D_{F}\right|^{1 / n(n-1)} A_{F} \leq 2^{7 / 6} B\left(\frac{1}{4}, \frac{1}{2}\right) .
$$

This bound is attained precisely when $F$ is a quartic form which, up to multiplication by a complex number, is equivalent under $G L_{2}(\mathbb{R})$ to $X Y\left(X^{2}-Y^{2}\right)$.

The approximate numerical value of $2^{7 / 6} B\left(\frac{1}{4}, \frac{1}{2}\right)$, and hence of $M_{4}$, is 11.7726. This compares with an approximate numerical value of 15.8997 for $M_{3}$.

Theorem 1 suggests that $M_{n}$ is attained precisely when $F$ is a form which, up to multiplication by a complex number, is $G L_{2}(\mathbb{R})$-equivalent to the form $F_{n}^{*}(X, Y)=\prod_{k=1}^{n}\left(X \sin \left(\frac{k \pi}{n}\right)-Y \cos \left(\frac{k \pi}{n}\right)\right)$. The next theorem reveals a striking correspondence between the collection of forms having a complete factorization over $\mathbb{R}$ and the set of equiangular polygons, which strongly suggests that $M_{n}=$ $D_{F_{n}^{*}}^{1 / n(n-1)} A_{F_{n}^{*}}$ and $\lim _{n \rightarrow \infty} M_{n}=2 \pi$. This correspondence, which arises from the Schwarz-Christoffel mapping formula, is interesting in its own right and may point to an even deeper connection between these algebraic and geometric quantities.

Theorem 2. There is a correspondence between the collection of forms $F$ of degree $n \geq 3$ having a complete factorization over $\mathbb{R}$ and the set of $n$-sided equiangular polygons such that the perimeter of the polygon corresponding to $F$ is $A_{F}$. Under this correspondence, $G L_{2}(\mathbb{R})$-equivalent forms are mapped to similar polygons, $S L_{2}(\mathbb{R})$-equivalent forms are mapped to congruent polygons, and forms equivalent to $F_{n}^{*}$ are mapped to $n$-sided regular polygons. Moreover, the correspondence is bijective when considered as a map between equivalence classes of forms and polygons.

From this correspondence, it follows that the limiting value of the sequence $D_{F_{n}^{*}}^{1 / n(n-1)} A_{F_{n}^{*}}$ is the circumference of some circle. Computational evidence suggests that this limiting value is $2 \pi$.

In the course of proving Theorem 2, we will actually show that the lengths of the sides of the equiangular polygon corresponding to $F$ are the areas of the sectors of $F$. (A sector of $F$ is a portion of the region $|F(x, y)| \leq 1$ bounded by two asymptotes of the curve $|F(x, y)|=1$ but containing none of the other asymptotes.) To be precise, let $F(X, Y)=\left(\alpha_{1} X-\beta_{1} Y\right) \cdots\left(\alpha_{n} X-\beta_{n} Y\right)$ where the $\alpha$ 's and the $\beta$ 's are real numbers, and suppose that the slopes of the asymptotes of the curve $|F(x, y)|=1$ are in the order

$$
\frac{\alpha_{1}}{\beta_{1}}<\frac{\alpha_{2}}{\beta_{2}}<\cdots<\frac{\alpha_{n}}{\beta_{n}}
$$

where possibly $\beta_{n}=0$. Further, let $A_{F}^{(k)}$ denote the area of the sector which is bounded by the asymptotes $\alpha_{k} x-\beta_{k} y=0$ and $\alpha_{k+1} x-\beta_{k+1} y=0$, but which does not contain any of the other asymptotes $\alpha_{s} x-\beta_{s} y=0, \quad s \neq k, k+1$. Then we will show that the lengths of the sides of the equiangular polygon 
corresponding to $F$ are, in counterclockwise order,

$$
A_{F}^{(1)}, A_{F}^{(2)}, \ldots, A_{F}^{(n)} \text {. }
$$

Since equiangular triangles are equilateral triangles and equiangular quadrilaterals are rectangles, this has the following consequence for cubic forms and quartic forms.

Corollary 2. If $F$ is a cubic form with real coefficients and positive discriminant, then each sector of $F$ has equal area. If $F$ is a quartic form with real coefficients for which $F(x, 1)$ has four distinct real roots, then alternating sectors have equal area.

Now an equivalence class (under congruence) of $n$-sided equiangular polygons is determined by $n-2$ independent geometric invariants - for example, by the length of one side and the ratio of this side to $n-3$ neighboring ones. Likewise, an equivalence class (under $S L_{2}(\mathbb{R})$ ) of binary forms of degree $n$ is determined by $n-2$ independent algebraic invariants. In fact, the space of algebraic invariants of a binary form $F$ of degree $n$ with a complete factorization over $\mathbb{R}$ has a basis which contains exactly $n-3$ absolute invariants and one non-absolute invariant (Salmon [14]).

The following representation theorem, which relies on the fact that the quantities $D_{F}^{1 / n(n-1)} A_{F}^{(k)}$ are absolutely invariant with respect to $G L_{2}(\mathbb{R})$, allows one to consider $D_{F}^{1 / n(n-1)} A_{F}$ (over the class of forms of degree $n$ with a complete factorization over $\mathbb{R}$ ) as a function of $n-3$ independent real variables on a bounded region. This representation is used in the proof of Theorem 1 to show that $M_{4}=D_{F_{4}^{*}}^{1 / 12} A_{F_{4}^{*}}$ and may be useful when trying to show that $M_{n}=D_{F_{n}^{*}}^{1 / n(n-1)} A_{F_{n}^{*}}$ in general.

Theorem 3. Let $F$ be a binary form of degree $n$ with real coefficients for which the polynomial $F(x, 1)$ has $n$ distinct real roots (counting any root at infinity) and suppose that the slopes of the asymptotes of the curve $|F(x, y)|=1$ are in the order

$$
\frac{\alpha_{1}}{\beta_{1}}<\frac{\alpha_{2}}{\beta_{2}}<\cdots<\frac{\alpha_{n}}{\beta_{n}}
$$

where possibly $\beta_{n}=0$. Let $A_{F}^{(k)}$ denote the area of the sector bounded by the asymptotes $\alpha_{k} x-\beta_{k} y=0$ and $\alpha_{k+1} x-\beta_{k+1} y=0$. Then

$$
D_{F}^{1 / n(n-1)} A_{F}^{(k)}=D_{F_{k}}^{1 / n(n-1)} \int_{0}^{1} \frac{d t}{F_{k}(1, t)^{2 / n}}
$$

and

where

$$
D_{F}^{1 / n(n-1)} A_{F}=\sum_{k=1}^{n} D_{F_{k}}^{1 / n(n-1)} \int_{0}^{1} \frac{d t}{F_{k}(1, t)^{2 / n}}
$$

and

$$
F_{k}(X, Y)=X Y(X-Y)\left(X-u_{1}^{(k)} Y\right) \cdots\left(X-u_{n-3}^{(k)} Y\right)
$$

$$
u_{j}^{(k)}=\frac{\left(\alpha_{k+1} \beta_{k}-\alpha_{k} \beta_{k+1}\right)\left(\alpha_{k-1} \beta_{k-1-j}-\alpha_{k-1-j} \beta_{k-1}\right)}{\left(\alpha_{k+1} \beta_{k-1}-\alpha_{k-1} \beta_{k+1}\right)\left(\alpha_{k} \beta_{k-1-j}-\alpha_{k-1-j} \beta_{k}\right)}
$$


for $1 \leq j \leq n-3,1 \leq k \leq n$, with the subscripts taken modulo $n$. For each $k$,

Moreover, if we put

$$
0<u_{1}^{(k)}<u_{2}^{(k)}<\cdots<u_{n-3}^{(k)}<1 .
$$

$$
u_{j}=u_{j}^{(1)}
$$

for $j=1,2, \ldots, n-3$, then the $u_{j}$ are independent absolute invariants and

$$
u_{j}^{(k)}=\frac{\left(u_{n-k}-u_{n-k-1}\right)\left(u_{n-k+1+j}-u_{n-k+1}\right)}{\left(u_{n-k+1}-u_{n-k-1}\right)\left(u_{n-k+1+j}-u_{n-k}\right)}
$$

provided that we adopt the convention that

$$
u_{0}=0, \quad u_{n-2}=1, \quad u_{n-1}=\infty \text {. }
$$

Hence, the quantity $D_{F}^{1 / n(n-1)} A_{F}$ may be considered a function of the invariants $u_{1}, \ldots, u_{n-3}$ over the region $0<u_{1}<\cdots<u_{n-3}<1$.

The integrals

$$
\int_{0}^{1} \frac{d t}{F_{k}(1, t)^{2 / n}}=\int_{0}^{1} \frac{d t}{t^{2 / n}(1-t)^{2 / n}\left(1-u_{1}^{(k)} t\right)^{2 / n} \cdots\left(1-u_{n-3}^{(k)} t\right)^{2 / n}}
$$

which arise in this representation are particular generalized hypergeometric functions. For quartic forms, these integrals reduce to ordinary hypergeometric functions (actually to elliptic integrals), and for cubic forms, they reduce to Beta functions.

In the notation of Theorem 3 , the form $F_{n}^{*}$ has absolute invariants

$$
u_{j}^{(k)}=u_{j}^{*}
$$

where

$$
u_{j}^{*}=\frac{1}{2}\left\{1-\frac{\tan \frac{\pi}{n}}{\tan (j+1) \frac{\pi}{n}}\right\}
$$

for $j=1,2, \ldots, n-3$. Let $Q_{n}$ denote the quantity $D_{F}^{1 / n(n-1)} A_{F}$ when it is considered as a function of the invariants $u_{1}, \ldots, u_{n-3}$ over the region $0<u_{1}<\cdots<u_{n-3}<1$. Theorem 1 will be proved by demonstrating that the function $Q_{4}$ has a unique maximum point at $u_{1}=u_{1}^{*}=\frac{1}{2}$. We believe that $\left(u_{1}^{*}, \ldots, u_{n-3}^{*}\right)$ is the unique maximum point of $Q_{n}$, in general, although we have been unable to demonstrate this for $n \geq 5$. We can prove a minor result in this direction.

Theorem 4. The point $\left(u_{1}^{*}, \ldots, u_{n-3}^{*}\right)$ defined by

$$
u_{j}^{*}=\frac{1}{2}\left\{1-\frac{\tan \frac{\pi}{n}}{\tan (j+1) \frac{\pi}{n}}\right\}
$$

for $1 \leq j \leq n-3$, is a critical point of the function $Q_{n}$ where

$$
Q_{n}\left(u_{1}, \ldots, u_{n-3}\right)=\sum_{k=1}^{n} D_{F_{k}}^{1 / n(n-1)} \int_{0}^{1} \frac{d t}{F_{k}(1, t)^{2 / n}}
$$

with the $F_{k}$ as in the statement of Theorem 3. 
A brief sketch of the proof of Theorem 4 will be given in $\S 6$ at the end of the paper.

\section{A REPRESENTATION FOR $D_{F}^{1 / n(n-1)} A_{F}$}

Let $F(X, Y)=\left(\alpha_{1} X-\beta_{1} Y\right) \cdots\left(\alpha_{n} X-\beta_{n} Y\right)$, where the $\alpha$ 's and the $\beta$ 's are real numbers, and suppose that the slopes of the asymptotes of the curve $|F(x, y)|=1$ are in the order

$$
\frac{\alpha_{1}}{\beta_{1}}<\frac{\alpha_{2}}{\beta_{2}}<\cdots<\frac{\alpha_{n}}{\beta_{n}}
$$

where possibly $\beta_{n}=0$. Let $A_{F}^{(k)}$ denote the area of the sector which is bounded by the asymptotes $\alpha_{k} x-\beta_{k} y=0$ and $\alpha_{k+1} x-\beta_{k+1} y=0$. We will show in this section that

$$
A_{F}^{(k)}=\frac{D_{F_{k}}^{1 / n(n-1)}}{D_{F}^{1 / n(n-1)}} \int_{0}^{1} \frac{d t}{F_{k}(1, t)^{2 / n}}
$$

where

$$
F_{k}(X, Y)=X Y(X-Y)\left(X-u_{1}^{(k)} Y\right) \cdots\left(X-u_{n-3}^{(k)} Y\right)
$$

and

$$
u_{j}^{(k)}=\frac{\left(\alpha_{k+1} \beta_{k}-\alpha_{k} \beta_{k+1}\right)\left(\alpha_{k-1} \beta_{k-1-j}-\alpha_{k-1-j} \beta_{k-1}\right)}{\left(\alpha_{k+1} \beta_{k-1}-\alpha_{k-1} \beta_{k+1}\right)\left(\alpha_{k} \beta_{k-1-j}-\alpha_{k-1-j} \beta_{k}\right)}
$$

for $j=1,2, \ldots, n-3$, with the subscripts taken modulo $n$. Theorem 3 will then follow.

In [2], we showed that the quantity $\left|D_{F}\right|^{1 / n(n-1)} A_{F}$ is absolutely invariant with respect to $G L_{2}(\mathbb{R})$ by observing that if $T=\left(\begin{array}{ll}a & b \\ c & d\end{array}\right) \in G L_{2}(\mathbb{R})$, then

$$
\begin{aligned}
A_{F} & =\iint_{|F(x, y)| \leq 1} d x d y \\
& =\iint_{|F(a u+b v, c u+d v)| \leq 1}|\operatorname{det} T| d u d v \\
& =|\operatorname{det} T| A_{F_{T}}
\end{aligned}
$$

and

$$
D_{F_{T}}=(\operatorname{det} T)^{n(n-1)} D_{F}
$$

We also showed that

$$
A_{F}=\int_{-\infty}^{\infty} \frac{d v}{|F(1, v)|^{2 / n}}
$$

using the fact that the curve $|F(x, y)|=1$ is symmetric about the origin and has the polar representation

$$
r=\frac{1}{|F(\cos \theta, \sin \theta)|^{1 / n}}
$$

In a similar fashion, one can demonstrate that $D_{F}^{1 / n(n-1)} A_{F}^{(k)}$ is an absolute invariant with respect to $G L_{2}(\mathbb{R})$ and that

$$
A_{F}^{(k)}=\int_{\alpha_{k} / \beta_{k}}^{\alpha_{k+1} / \beta_{k+1}} \frac{d v}{|F(1, v)|^{2 / n}}
$$


for $k=1, \ldots, n-1$, while

$$
A_{F}^{(n)}=\int_{-\infty}^{\alpha_{1} / \beta_{1}} \frac{d v}{|F(1, v)|^{2 / n}}+\int_{\alpha_{n} / \beta_{n}}^{\infty} \frac{d v}{|F(1, v)|^{2 / n}}
$$

The only additional observation which needs to be made to demonstrate the absolute invariance of $D_{F}^{1 / n(n-1)} A_{F}^{(k)}$ is that if $T=\left(\begin{array}{ll}a & b \\ c & d\end{array}\right) \in G L_{2}(\mathbb{R})$, then every sector of $F$ is mapped to a sector of $F_{T}$. It is certainly true that an asymptote of the curve $|F(x, y)|=1$ is mapped to an asymptote of the curve $\left|F_{T}(x, y)\right|=1$. Further, since the slopes of the asymptotes are transformed according to the rule

$$
t \longmapsto \frac{a t-c}{d-b t},
$$

the clockwise order of the asymptotes is either preserved or reversed. Hence every sector of $F$ is mapped to a region which is bounded by the curve $\left|F_{T}(x, y)\right|$ $=1$ and two of its asymptotes, and which does not contain any other asymptotes; that is, every sector of $F$ is mapped to a sector of $F_{T}$.

The representation of $A_{F}^{(k)}$ which we will derive below relies on the absolute invariance of $D_{F}^{1 / n(n-1)} A_{F}^{(k)}$. In the argument which follows, we will appeal to the fact that a linear substitution applied to the form $F$ induces a fractional linear transformation of the roots of $F(1, y)$ and that any such transformation having real coefficients may be defined by the rule

$$
\frac{\left(w-w_{1}\right)\left(w_{3}-w_{2}\right)}{\left(w-w_{2}\right)\left(w_{3}-w_{1}\right)}=\frac{\left(z-z_{1}\right)\left(z_{3}-z_{2}\right)}{\left(z-z_{2}\right)\left(z_{3}-z_{1}\right)}
$$

where the $z$ 's and the $w$ 's are real numbers such that $z_{1}, z_{2}, z_{3}$ are mapped to $w_{1}, w_{2}, w_{3}$ respectively.

To simplify notation, let us assume that $k=1$. Hence consider the sector which is bounded by the asymptotes $\alpha_{1} x-\beta_{1} y=0$ and $\alpha_{2} x-\beta_{2} y=0$. There is a linear substitution with real coefficients such that

$$
\frac{\alpha_{1}}{\beta_{1}} \longmapsto 0, \frac{\alpha_{2}}{\beta_{2}} \longmapsto 1, \frac{\alpha_{n}}{\beta_{n}} \longmapsto \infty
$$

Under such a substitution, $F$ is transformed to the form

$$
m X Y(X-Y)\left(X-u_{1} Y\right) \cdots\left(X-u_{n-3} Y\right)
$$

where $0<u_{1}<u_{2}<\cdots<u_{n-3}<1$ and $m \in \mathbb{R}$. The latter form is equivalent under $G L_{2}(\mathbb{R})$ to at least one of the forms

$$
\pm X Y(X-Y)\left(X-u_{1} Y\right) \cdots\left(X-u_{n-3} Y\right)
$$

(determined by the sign of $m$ ). Hence, putting

$$
F_{1}(X, Y)=X Y(X-Y)\left(X-u_{1} Y\right) \cdots\left(X-u_{n-3} Y\right),
$$


it follows, by the invariance of $D_{F}^{1 / n(n-1)} A_{F}^{(1)}$, that

$$
\begin{aligned}
A_{F}^{(1)} & =\frac{D_{F_{1}}^{1 / n(n-1)}}{D_{F}^{1 / n(n-1)}} \int_{0}^{1} \frac{d t}{F_{1}(1, t)^{2 / n}} \\
& =\frac{D_{F_{1}}^{1 / n(n-1)}}{D_{F}^{1 / n(n-1)}} \int_{0}^{1} \frac{d t}{t^{2 / n}(1-t)^{2 / n}\left(1-u_{1} t\right)^{2 / n} \cdots\left(1-u_{n-3} t\right)^{2 / n}} .
\end{aligned}
$$

The quantities $u_{1}, u_{2}, \ldots, u_{n-3}$ can be explicitly determined in the following way. First, observe that since $\alpha_{1} / \beta_{1}, \alpha_{2} / \beta_{2}, \alpha_{n} / \beta_{n}$ are mapped to 0,1 , $\infty$ respectively, the slopes of the asymptotes are transformed according to the fractional linear transformation

$$
t \longmapsto \frac{\left(\beta_{1} t-\alpha_{1}\right)\left(\alpha_{2} \beta_{n}-\alpha_{n} \beta_{2}\right)}{\left(\beta_{n} t-\alpha_{n}\right)\left(\alpha_{2} \beta_{1}-\alpha_{1} \beta_{2}\right)} .
$$

In particular,

$$
\frac{\alpha_{i}}{\beta_{i}} \longmapsto \frac{\left(\beta_{1} \alpha_{i}-\alpha_{1} \beta_{i}\right)\left(\alpha_{2} \beta_{n}-\alpha_{n} \beta_{2}\right)}{\left(\beta_{n} \alpha_{i}-\alpha_{n} \beta_{i}\right)\left(\alpha_{2} \beta_{1}-\alpha_{1} \beta_{2}\right)} .
$$

Further, such a transformation preserves the clockwise order of the slopes. Now the curve $\left|F_{1}(x, y)\right|=1$ has asymptotes whose slopes are in the order

$$
0<1<\frac{1}{u_{n-3}}<\cdots<\frac{1}{u_{1}}<\infty
$$

Hence, we have

$$
u_{j}=\frac{\left(\alpha_{n-j} \beta_{n}-\alpha_{n} \beta_{n-j}\right)\left(\alpha_{2} \beta_{1}-\alpha_{1} \beta_{2}\right)}{\left(\alpha_{n-j} \beta_{1}-\alpha_{1} \beta_{n-j}\right)\left(\alpha_{2} \beta_{n}-\alpha_{n} \beta_{2}\right)}
$$

for $j=1,2, \ldots, n-3$. Notice that the $u_{j}$ are independent absolute invariants, and that, in fact, each $u_{j}$ is a particular cross ratio of the slopes of the asymptotes of the curve $|F(x, y)|=1$.

In a similar fashion, we have

$$
\begin{aligned}
A_{F}^{(k)} & =\frac{D_{F_{k}}^{1 / n(n-1)}}{D_{F}^{1 / n(n-1)}} \int_{0}^{1} \frac{d t}{F_{k}(1, t)^{2 / n}} \\
& =\frac{D_{F_{k}}^{1 / n(n-1)}}{D_{F}^{1 / n(n-1)}} \int_{0}^{1} \frac{d t}{t^{2 / n}(1-t)^{2 / n}\left(1-u_{1}^{(k)} t\right)^{2 / n} \cdots\left(1-u_{n-3}^{(k)} t\right)^{2 / n}}
\end{aligned}
$$

for $k=1,2, \ldots, n$, where the $F_{k}$ and $u_{j}^{(k)}$ are as defined above. In particular,

$$
0<u_{1}^{(k)}<u_{2}^{(k)}<\cdots<u_{n-3}^{(k)}<1 .
$$

Notice that if we put $u_{0}^{(k)}=0, u_{n-2}^{(k)}=1$, then the $u_{j}^{(k)}$ satisfy the relations

$$
u_{1}^{(k)}=u_{j}^{(k)}\left(1-u_{j-1}^{(k-1)}\right)
$$

for $1 \leq j \leq n-2,1 \leq k \leq n$ (subscripts and superscripts taken modulo $n$ ). The $u_{j}^{(k)}$ are actually certain cross ratios of the invariants $u_{1}, u_{2}, \ldots, u_{n-3}$. 
To be specific,

$$
u_{j}^{(k)}=\frac{\left(u_{n-k}-u_{n-k-1}\right)\left(u_{n-k+1+j}-u_{n-k+1}\right)}{\left(u_{n-k+1}-u_{n-k-1}\right)\left(u_{n-k+1+j}-u_{n-k}\right)} .
$$

This follows from the observation that $F_{k}$ is equivalent under $G L_{2}(\mathbb{R})$ to one of the forms $\pm X Y(X-Y)\left(X-u_{1} Y\right) \cdots\left(X-u_{n-3} Y\right)$.

Combining these expressions for $A_{F}^{(k)}$, we have

$$
\begin{aligned}
D_{F}^{1 / n(n-1)} A_{F} & =D_{F}^{1 / n(n-1)} \sum_{k=1}^{n} A_{F}^{(k)} \\
& =\sum_{k=1}^{n} D_{F_{k}}^{1 / n(n-1)} \int_{0}^{1} \frac{d t}{F_{k}(1, t)^{2 / n}} .
\end{aligned}
$$

In particular, the quantity $D_{F}^{1 / n(n-1)} A_{F}$ may be considered as a function of the $n-3$ independent absolute invariants $u_{1}, u_{2}, \ldots, u_{n-3}$ over the bounded region

$$
0<u_{1}<u_{2}<\cdots<u_{n-3}<1 .
$$

Theorem 3 now follows.

\section{QUARTIC FORMS: ProOF OF TheOReM 1}

In this section, we will show that $M_{4}=2^{7 / 6} B\left(\frac{1}{4}, \frac{1}{2}\right)$ and that this value is attained precisely for quartic forms which, up to multiplication by a complex number, are equivalent under $G L_{2}(\mathbb{R})$ to $F_{4}^{*}(X, Y)=\frac{1}{2} X Y\left(X^{2}-Y^{2}\right)$.

Since $\left|D_{F}\right|^{1 / 12} A_{F}<M_{4}$ if $F$ is quartic form for which $F(x, 1)$ has at least one non-real root, and since $\left|D_{F}\right|^{1 / 12} A_{F}$ is invariant with respect to replacing $F$ by $k F$ for any complex number $k$, we may restrict our attention to the quartic forms with real coefficients for which $F(x, 1)$ has four distinct real roots (counting any root at infinity). By the theorem of the previous section, if $F(X, Y)=\left(\alpha_{1} X-\beta_{1} Y\right)\left(\alpha_{2} X-\beta_{2} Y\right)\left(\alpha_{3} X-\beta_{3} Y\right)\left(\alpha_{4} X-\beta_{4} Y\right)$ where the $\alpha$ 's and the $\beta$ 's are real numbers with

$$
\frac{\alpha_{1}}{\beta_{1}}<\frac{\alpha_{2}}{\beta_{2}}<\frac{\alpha_{3}}{\beta_{3}}<\frac{\alpha_{4}}{\beta_{4}},
$$

then

$$
\begin{aligned}
D_{F}^{1 / 12} A_{F}= & 2 u^{1 / 6}(1-u)^{1 / 6} \int_{0}^{1} \frac{d t}{t^{1 / 2}(1-t)^{1 / 2}(1-u t)^{1 / 2}} \\
& +2 u^{1 / 6}(1-u)^{1 / 6} \int_{0}^{1} \frac{d t}{t^{1 / 2}(1-t)^{1 / 2}(1-(1-u) t)^{1 / 2}}
\end{aligned}
$$

where

$$
u=\frac{\left(\alpha_{2} \beta_{1}-\alpha_{1} \beta_{2}\right)\left(\alpha_{4} \beta_{3}-\alpha_{3} \beta_{4}\right)}{\left(\alpha_{2} \beta_{4}-\alpha_{4} \beta_{2}\right)\left(\alpha_{1} \beta_{3}-\alpha_{3} \beta_{1}\right)}
$$




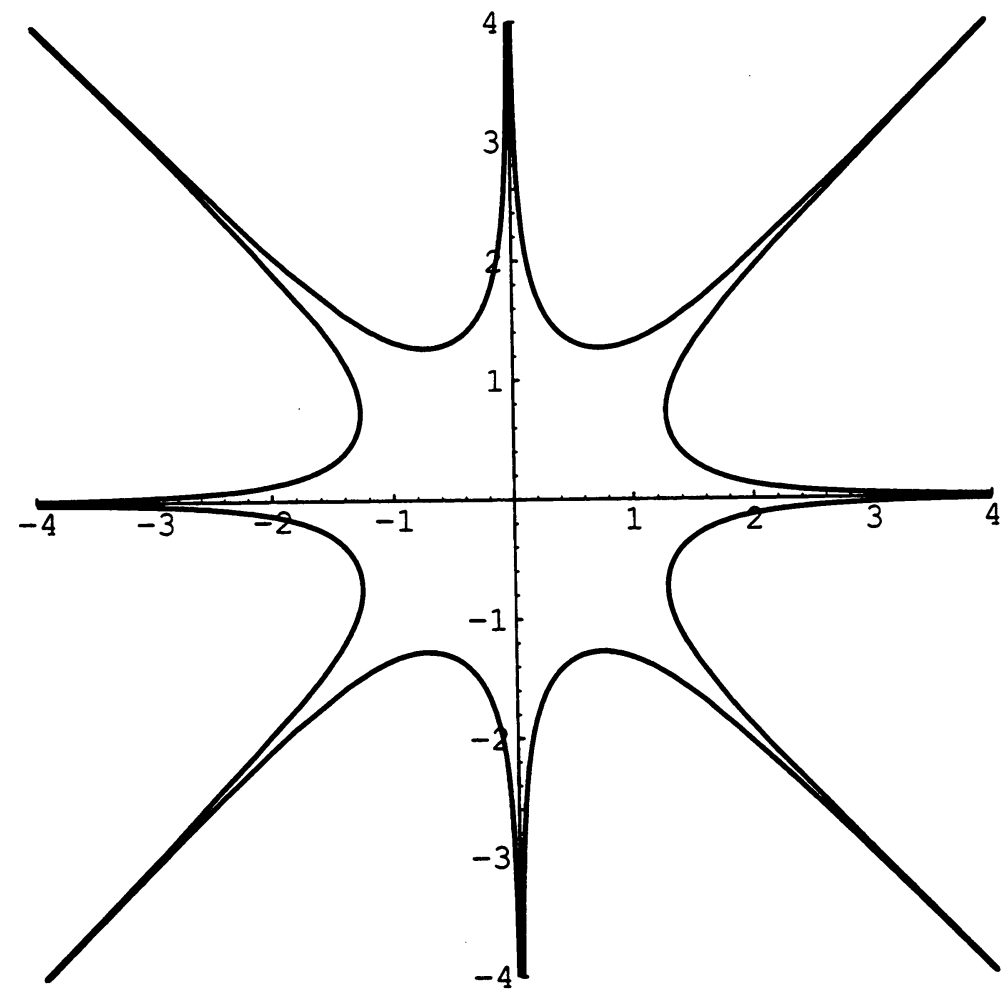

FIGURE 3. $\left|x y\left(x^{2}-y^{2}\right)\right|=1$

In particular, $F_{4}^{*}$ has absolute invariant $u^{*}=\frac{1}{2}$. Further, if $F(X, Y)=$ $X Y\left(X^{2}-Y^{2}\right)$, then

$$
\begin{aligned}
D_{F}^{1 / 12} A_{F} & =D_{F}^{1 / 12} \int_{-\infty}^{\infty} \frac{d v}{|F(1, v)|^{1 / 2}} \\
& =4 D_{F}^{1 / 12} \int_{0}^{1} \frac{d v}{v^{1 / 2}\left(1-v^{2}\right)^{1 / 2}} \\
& =2 D_{F}^{1 / 12} \int_{0}^{1} \frac{d z}{z^{3 / 4}(1-z)^{1 / 2}} \\
& =2^{7 / 6} B\left(\frac{1}{4}, \frac{1}{2}\right) .
\end{aligned}
$$

Hence, to prove Theorem 1, it suffices to demonstrate that the expression

$$
\begin{aligned}
u^{1 / 6}(1-u)^{1 / 6} & \int_{0}^{1} \frac{d t}{t^{1 / 2}(1-t)^{1 / 2}(1-u t)^{1 / 2}} \\
& +u^{1 / 6}(1-u)^{1 / 6} \int_{0}^{1} \frac{d t}{t^{1 / 2}(1-t)^{1 / 2}(1-(1-u) t)^{1 / 2}},
\end{aligned}
$$

where $u \in(0,1)$, is maximized precisely for $u=\frac{1}{2}$. 


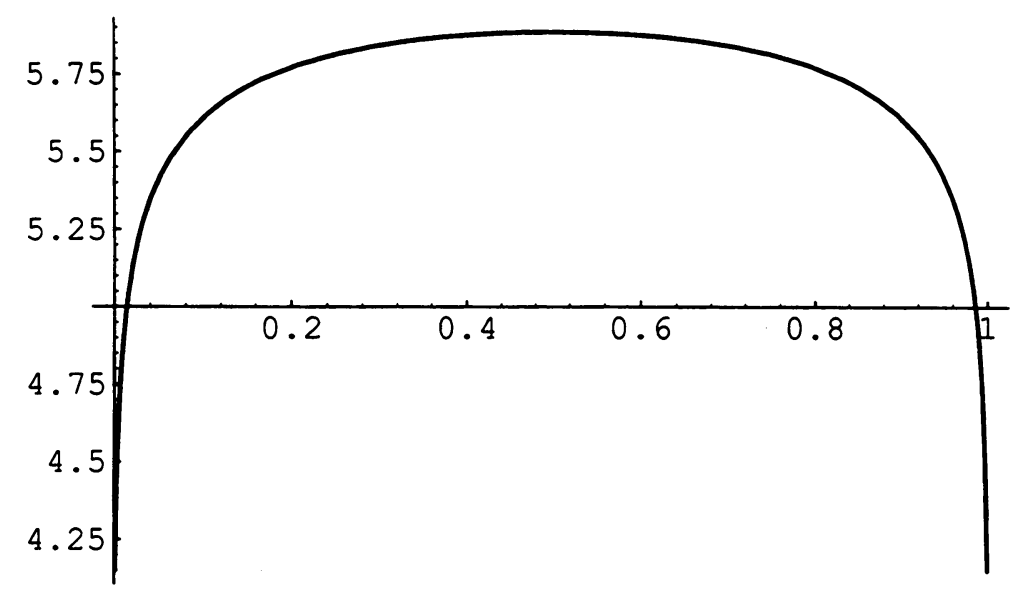

FIGURE 4. Graph of $N$

Put

$$
\begin{aligned}
& G(x)=\int_{0}^{1} \frac{d t}{t^{1 / 2}(1-t)^{1 / 2}(1-x t)^{1 / 2}}, \\
& L(x)=x^{1 / 6}(1-x)^{1 / 6},
\end{aligned}
$$

and let $N$ be defined by

$$
N(x)=L(x) G(x)+L(1-x) G(1-x)
$$

for $x \in(0,1)$. Notice that $N$ is symmetric about $x=\frac{1}{2}$ (Figure 4). Using the estimates $1-x \leq 1-x t$ and $1-t \leq 1-x t$, we have

$$
\begin{aligned}
L(x) G(x) & \leq x^{1 / 6}(1-x)^{1 / 12} \int_{0}^{1} \frac{(1-x t)^{1 / 12} d t}{t^{1 / 2}(1-t)^{1 / 2}(1-x t)^{1 / 2}} \\
& \leq x^{1 / 6}(1-x)^{1 / 12} \int_{0}^{1} \frac{d t}{t^{1 / 2}(1-t)^{11 / 12}} \\
& =x^{1 / 6}(1-x)^{1 / 12} B\left(\frac{1}{2}, \frac{1}{12}\right)
\end{aligned}
$$

and so $L(x) G(x) \rightarrow 0$ as $x \rightarrow 1$. Similarly, $L(x) G(x) \rightarrow 0$ as $x \rightarrow 0$. Hence $N$ tends to zero at the endpoints of the interval $(0,1)$. From the symmetry of $N$, it is clear that $x=\frac{1}{2}$ is a critical point of $N$. We will show that $N^{\prime}(x)>0$ for $x<\frac{1}{2}$ and $N^{\prime}(x)<0$ for $x>\frac{1}{2}$, and it will follow that $x=\frac{1}{2}$ is the unique maximal point of $N$.

Now the function $G$ is the integral representation of a particular hypergeometric function. The general hypergeometric function is defined by the series

$$
F(a, b ; c ; x)=\sum_{n=0}^{\infty} \frac{(a)_{n}(b)_{n}}{(c)_{n}} \frac{x^{n}}{n !}, \quad|x|<1,
$$

where

$$
(\lambda)_{n}=\frac{\Gamma(\lambda+n)}{\Gamma(\lambda)}
$$


and has the integral representation

$$
F(a, b ; c ; x)=\frac{\Gamma(c)}{\Gamma(b) \Gamma(c-b)} \int_{0}^{1} t^{b-1}(1-t)^{c-b-1}(1-x t)^{-a} d t
$$

for $c>b>0$; moreover, this function satisfies the differential equation

$$
x(1-x) \frac{d^{2} w}{d x^{2}}+(c-(a+b+1) x) \frac{d w}{d x}-a b w=0
$$

(see Abramowitz and Stegun [1]). Hence

$$
G(x)=\pi F\left(\frac{1}{2}, \frac{1}{2} ; 1 ; x\right)
$$

and $G$ satisfies the differential equation

$$
x(1-x) G^{\prime \prime}(x)+(1-2 x) G^{\prime}(x)-\frac{1}{4} G(x)=0 .
$$

Further, $L$ satisfies the differential equation

$$
6 x(1-x) L^{\prime}(x)-(1-2 x) L(x)=0 .
$$

Put

$$
M(x)=\frac{6 x(1-x)}{L(x) L(1-x)} N^{\prime}(x) .
$$

We claim that $M^{\prime}(x)<0$. To see this, notice that

$$
N^{\prime}(x)=L^{\prime}(x) G(x)+L(x) G^{\prime}(x)-L^{\prime}(1-x) G(1-x)-L(1-x) G^{\prime}(1-x)
$$

and so, using the equation for $L$, we have

$$
\begin{aligned}
M(x)=(1 & -2 x)\left\{\frac{G(x)}{L(1-x)}+\frac{G(1-x)}{L(x)}\right\} \\
& +6 x(1-x)\left\{\frac{G^{\prime}(x)}{L(1-x)}-\frac{G^{\prime}(1-x)}{L(x)}\right\}
\end{aligned}
$$

Differentiating the two expressions on the right-hand side of this equation, and using the equation for $L$ again, we have

$$
\begin{aligned}
\frac{d}{d x}(1-2 x) & \left\{\frac{G(x)}{L(1-x)}+\frac{G(1-x)}{L(x)}\right\} \\
=-2 & \left\{\frac{G(x)}{L(1-x)}+\frac{G(1-x)}{L(x)}\right\} \\
+ & (1-2 x)\left\{\frac{G^{\prime}(x)}{L(1-x)}-\frac{G^{\prime}(1-x)}{L(x)}\right\} \\
- & \frac{(1-2 x)^{2}}{6 x(1-x)}\left\{\frac{G(x)}{L(1-x)}+\frac{G(1-x)}{L(x)}\right\}
\end{aligned}
$$


and

$$
\begin{aligned}
\frac{d}{d x} 6 x(1-x) & \left\{\frac{G^{\prime}(x)}{L(1-x)}-\frac{G^{\prime}(1-x)}{L(x)}\right\} \\
=6(1-2 x) & \left\{\frac{G^{\prime}(x)}{L(1-x)}-\frac{G^{\prime}(1-x)}{L(x)}\right\} \\
+ & 6 x(1-x)\left\{\frac{G^{\prime \prime}(x)}{L(1-x)}+\frac{G^{\prime \prime}(1-x)}{L(x)}\right\} \\
& -(1-2 x)\left\{\frac{G^{\prime}(x)}{L(1-x)}-\frac{G^{\prime}(1-x)}{L(x)}\right\}
\end{aligned}
$$

Using the equation for $G$, we have

$$
\begin{gathered}
6 x(1-x)\left\{\frac{G^{\prime \prime}(x)}{L(1-x)}+\frac{G^{\prime \prime}(1-x)}{L(x)}\right\}+6(1-2 x)\left\{\frac{G^{\prime}(x)}{L(1-x)}-\frac{G^{\prime}(1-x)}{L(x)}\right\} \\
=\frac{3}{2}\left\{\frac{G(x)}{L(1-x)}+\frac{G(1-x)}{L(x)}\right\} .
\end{gathered}
$$

Hence, we find that

$$
M^{\prime}(x)=-\left\{\frac{1}{2}+\frac{(1-2 x)^{2}}{6 x(1-x)}\right\}\left\{\frac{G(x)}{L(1-x)}+\frac{G(1-x)}{L(x)}\right\}
$$

and so $M^{\prime}(x)<0$ as claimed.

Now $M^{\prime}(x)<0$ means that $M$ is decreasing. Since $M$ and $N^{\prime}$ have the same sign, and since $N^{\prime}\left(\frac{1}{2}\right)=0$, it follows that $N^{\prime}(x)>0$ for $0<x<\frac{1}{2}$ and $N^{\prime}(x)<0$ for $\frac{1}{2}<x<1$. Hence, $x=\frac{1}{2}$ is the only critical point of $N$, and this critical point is the maximum point. Theorem 1 now follows.

It is worth pointing out that Theorem 1 cannot be proved by examining the second derivative of $N$ directly. It was for this reason that the function $M$ was introduced. Unfortunately, the arguments of this section do not generalize, in an obvious way, to the functions $Q_{n}$ given by

$$
Q_{n}\left(u_{1}, \ldots, u_{n-3}\right)=\sum_{k=1}^{n} D_{F_{k}}^{1 / n(n-1)} \int_{0}^{1} \frac{d t}{F_{k}(1, t)^{2 / n}}
$$

(where the $F_{k}$ are as in the statement of Theorem 3).

\section{The Schwarz-Christoffel correspondence: Proof of Theorem 2}

In this section, we will explain how the correspondence between forms and polygons described in the statement of Theorem 2 arises from the SchwarzChristoffel transformations. We will also discuss the implications of this correspondence in greater detail.

Recall that the Schwarz-Christoffel transformations are analytic functions which map the upper half of the complex plane conformally onto the interior of a polygon in a particular way. Named after the German mathematicians Hermann A. Schwarz (1843-1921) and Elwin B. Christoffel (1829-1900), who 
independently discovered them, these maps have important applications in electrostatics and the study of fluids. The general form of the transformation is

$$
f(z)=A \int_{z_{0}}^{z} \frac{d s}{\left(s-s_{1}\right)^{k_{1}} \cdots\left(s-s_{n}\right)^{k_{n}}}+B
$$

where the $s_{j}$ are arbitrary real numbers with

$$
s_{1}<s_{2}<\cdots<s_{n},
$$

the $k_{j}$ are positive real numbers with

$$
k_{1}+k_{2}+\cdots+k_{n}=2,
$$

and $A, B, z_{0}$ are complex numbers with

$$
z_{0} \in\left\{z \in \mathbb{C}: \Im z \geq 0, z \neq s_{j}(j=1, \ldots, n)\right\} ;
$$

it is assumed that the factors $\left(s-s_{j}\right)^{k_{j}}$ represent branches of power functions with branch cuts extending below the real axis. Such a function $f$ is analytic on $\left\{z \in \mathbb{C}: \Im z \geq 0, z \neq s_{j}\right\}$, continuous on $\{z \in \mathbb{C}: \Im z \geq 0\}$, and maps the real axis to the boundary of a convex polygon whose exterior angles, in counterclockwise order, are $k_{1} \pi, k_{2} \pi, \ldots, k_{n} \pi$.

It is not difficult to show that the image of such an $f$ is an $n$-sided polygon, if we assume $a$ priori that $f$ is analytic on $\left\{z \in \mathbb{C}: \Im z \geq 0, z \neq s_{j}\right\}$. For then, using (2) we have the formula

$$
f^{\prime}(z)=A\left(z-s_{1}\right)^{-k_{1}} \cdots\left(z-s_{n}\right)^{-k_{n}}
$$

from which we can determine the image of the real axis. To be more specific, let us consider the line segment joining $s_{j}$ and $s_{j+1}$. Suppose that $\gamma(t)$ is a parametrization of this segment with $\gamma^{\prime}(t)>0$, and that $\omega(t)=f(\gamma(t))$ is the corresponding parametrization of its image. Then $\arg \omega^{\prime}(t)=\arg f^{\prime}(\gamma(t)) \gamma^{\prime}(t)=$ $\arg f^{\prime}(\gamma(t))$, and so the direction of the image curve is determined by the values of $\arg f^{\prime}(z)$. However from (3), we have

$$
\arg f^{\prime}(z)=\arg A-k_{1} \arg \left(z-s_{1}\right)-\cdots-k_{n} \arg \left(z-s_{n}\right)
$$

which is constant for $z \in\left(s_{j}, s_{j+1}\right)$. Hence, the image of the interval $\left(s_{j}, s_{j+1}\right)$ is the straight line segment joining $f\left(s_{j}\right)$ and $f\left(s_{j+1}\right)$. Now, as $z$ crosses the point $s_{j}$ from the left, the argument of $f^{\prime}(z)$ jumps by $k_{j} \pi$; hence $f\left(s_{j}\right)$ is a vertex with exterior angle $k_{j} \pi$. (See Figure 5.) Since $k_{1}+\cdots+k_{n}=2$, and $k_{j}>0$ for all $j$, it follows that the image of the real axis under $f$ is the boundary of a convex $n$-sided polygon.

It is more difficult to find the Schwarz-Christoffel transformation $f$ whose image is a given polygon. From the preceding paragraph, it is clear that the numbers $k_{j}$ are determined (up to a cyclic permutation) by the exterior angles of the image polygon. It is also clear that the numbers $A$ and $B$ depend only on the position and size of the image polygon (since the effect of the complex map $z \mapsto A z+B$ on any region in the plane is to magnify by $|A|$, rotate by $\arg A$, and translate by $B$ ), and can be easily determined once a Schwarz-Christoffel map with image polygon similar to the given polygon is known. Hence the greatest difficulty rests with determining the numbers $s_{j}$. 

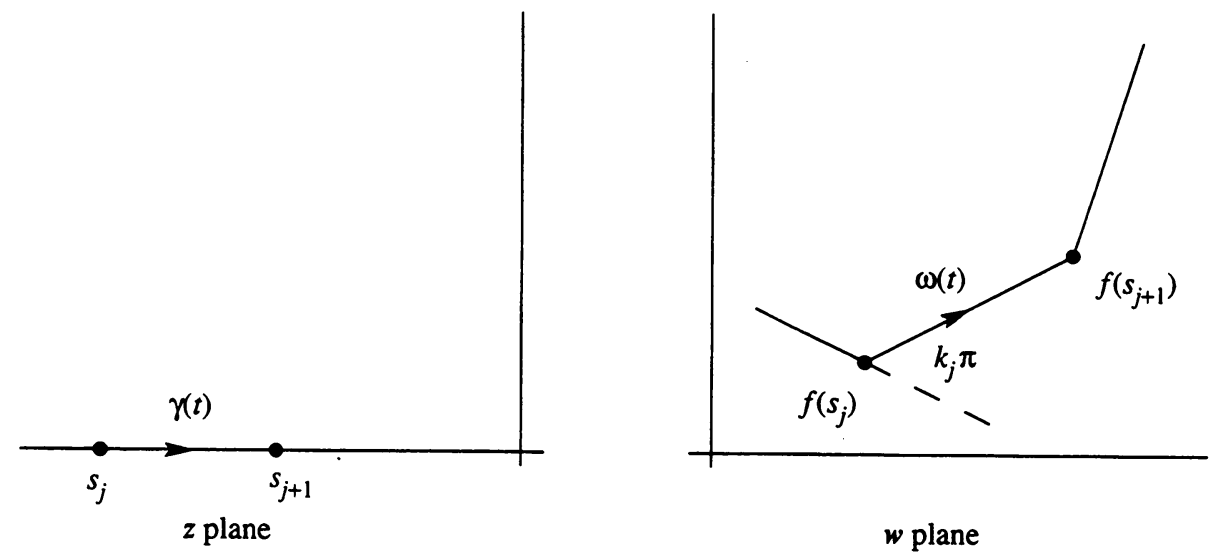

FIGURE 5. The action of $f$ on $\left(s_{j}, s_{j+1}\right)$

Now

$$
f\left(s_{j+1}\right)-f\left(s_{j}\right)=A \int_{s_{j}}^{s_{j+1}} \frac{d s}{\left(s-s_{1}\right)^{k_{1}} \cdots\left(s-s_{n}\right)^{k_{n}}} ;
$$

so the length of the side corresponding to the interval $\left(s_{j}, s_{j+1}\right)$ is

$$
|A| \int_{s_{j}}^{s_{j+1}} \frac{d s}{\left|s-s_{1}\right|^{k_{1}} \cdots\left|s-s_{n}\right|^{k_{n}}}
$$

Further, since an $n$-sided polygon with fixed exterior angles is determined up to similarity by the ratios of the lengths of $n-2$ consecutive sides, it follows that the $s_{j}$ must satisfy the $n-3$ equations

$$
\int_{s_{j}}^{s_{j+1}} \frac{d s}{\left|s-s_{1}\right|^{k_{1}} \cdots\left|s-s_{n}\right|^{k_{n}}}=c_{j} \int_{s_{j+1}}^{s_{j+2}} \frac{d s}{\left|s-s_{1}\right|^{k_{1}} \cdots\left|s-s_{n}\right|^{k_{n}}}
$$

$(j=1, \ldots, n-3)$ where the $c_{j}$ are numbers determined by the side lengths of the given polygon. Consequently, to determine values for the $s_{j}$, we need only specify three of them and then use the equations (4) to determine the rest. (For a complete discussion of the Schwarz-Christoffel transformations, see [7].)

We now proceed to describe the correspondence of Theorem 2 . Let $F$ be a form of degree $n \geq 3$ having a complete factorization over $\mathbb{R}$, and suppose that $F$ has the factorization

$$
F(X, Y)=a\left(Y-s_{1} X\right)\left(Y-s_{2} X\right) \cdots\left(Y-s_{n} X\right)
$$

with $s_{1}<s_{2}<\cdots<s_{n}$. We associate with $F$ the $n$-sided equiangular polygon $P(F)$ which is the image of the upper half plane under the Schwarz-Christoffel 
map

$$
g(z)=\frac{1}{a^{2 / n}} \int_{0}^{z} \frac{d s}{\left(s-s_{1}\right)^{2 / n} \cdots\left(s-s_{n}\right)^{2 / n}} .
$$

(When $F$ has a factor of $X$, we take $s_{n}=\infty$ and drop the factor $\left(s-s_{n}\right)^{2 / n}$ from the integral.)

From our discussion of the Schwarz-Christoffel transformations, it is clear that the vertices of $P(F)$ are, in counterclockwise order,

$$
g\left(s_{1}\right), g\left(s_{2}\right), \ldots, g\left(s_{n}\right),
$$

and that the exterior angles of $P(F)$ are all equal to $2 \pi / n$. It is also clear that the length of the side with vertices $g\left(s_{k}\right)$ and $g\left(s_{k+1}\right)$ is

$$
L_{P(F)}^{(k)}=\int_{s_{k}}^{s_{k+1}} \frac{d s}{\left|a\left(s-s_{1}\right) \cdots\left(s-s_{n}\right)\right|^{2 / n}}
$$

$(k=1,2, \ldots, n-1)$ and that the length of the side with vertices $g\left(s_{n}\right)$ and $g\left(s_{1}\right)$ is

$$
L_{P(F)}^{(n)}=\int_{-\infty}^{s_{1}} \frac{d s}{\left|a\left(s-s_{1}\right) \cdots\left(s-s_{n}\right)\right|^{2 / n}}+\int_{s_{n}}^{\infty} \frac{d s}{\left|a\left(s-s_{1}\right) \cdots\left(s-s_{n}\right)\right|^{2 / n}} .
$$

However, from $\S 3$, we recognize this expression as $A_{F}^{(k)}$, the area of the sector of $F$ bounded by the asymptotes $y-s_{k} x=0$ and $y-s_{k+1} x=0$. Hence the perimeter of the polygon $P(F)$ is

$$
\begin{aligned}
L_{P(F)} & =\sum_{k=1}^{n} L_{P(F)}^{(k)} \\
& =\int_{-\infty}^{\infty} \frac{d s}{\left|a\left(s-s_{1}\right) \cdots\left(s-s_{n}\right)\right|^{2 / n}} \\
& =A_{F} .
\end{aligned}
$$

(See Table 1 on next page.)

The correspondence defined through (5) also has the property that $F$ and $G$ are $S L_{2}(\mathbb{R})$-equivalent forms if and only if $P(F)$ and $P(G)$ are congruent polygons, and the property that $F$ and $G$ are $G L_{2}(\mathbb{R})$-equivalent forms if and only if $P(F)$ and $P(G)$ are similar. To see this, suppose that $F$ and $G$ are equivalent under $G L_{2}(\mathbb{R})$. Then since the quantities $\left|D_{F}\right|^{1 / n(n-1)} A_{F}^{(k)}$ are absolute invariants, there is a constant $c$ and an integer $l \in[0, n-1]$ such that $A_{F}^{(k)}=c A_{G}^{(k+l)}$ for $k=1,2, \ldots, n$. From the previous paragraph, this means that $L_{P(F)}^{(k)}=c L_{P(G)}^{(k+l)}$ for $k=1,2, \ldots, n$; hence $P(F)$ and $P(G)$ are similar polygons. (Note that if $F$ and $G$ are $S L_{2}(\mathbb{R})$-equivalent then the constant $c$ is necessarily one, and so $P(F)$ and $P(G)$ are congruent.) On the other hand, suppose that $F$ and $G$ are not equivalent under $G L_{2}(\mathbb{R})$. Then by (4), the sequence of ratios $A_{F}^{(k+1)} / A_{F}^{(k)}$ is not a cyclic rearrangement of the sequence $A_{G}^{(k+1)} / A_{G}^{(k)}$. Hence there is no $l$ such that $L_{P(F)}^{(k+1)} / L_{P(F)}^{(k)}=L_{P(G)}^{(k+l+1)} / L_{P(G)}^{(k+l)}$ for all $k$, and so $P(F)$ and $P(G)$ cannot be similar. In particular, if $F$ and 
TABLE 1. Summary of the correspondence

\begin{tabular}{|c|c|}
\hline $\begin{array}{c}\text { Forms } F \text { of degree } n \text { having } \\
\text { a complete factorization over } \mathbb{R}\end{array}$ & $n$-sided equiangular polygons $P$ \\
\hline area $A_{F}$ & perimeter $L_{P}$ \\
sector areas $A_{F}^{(k)}$ & side lengths $L_{P}^{(k)}$ \\
special form $F_{n}^{*}$ & $\begin{array}{c}n \text {-sided regular polygon } \\
\text { with perimeter } A_{F_{n}^{*}} \\
\text { equivalence under } G L_{2}(\mathbb{R}) \\
\text { equivalence under } S L_{2}(\mathbb{R})\end{array}$ \\
equivalence under similarity \\
equivalence under congruence \\
quartic forms
\end{tabular}

$G$ are not equivalent under $S L_{2}(\mathbb{R})$, then $P(F)$ and $P(G)$ are not congruent. We conclude that the correspondence between forms and polygons defined through (5) becomes bijective when considered as a correspondence between equivalence classes of forms and polygons.

Now the form

$$
F_{n}^{*}(X, Y)=\prod_{k=1}^{n}\left(X \sin \left(\frac{k \pi}{n}\right)-Y \cos \left(\frac{k \pi}{n}\right)\right)
$$

has the property that each of its sectors has equal area. Indeed, the curve $\left|F_{n}^{*}(x, y)\right|=1$ is invariant with respect to rotations of $\frac{\pi}{n}$ radians (see Figure 6). Hence, every form equivalent to $F_{n}^{*}$ is mapped to a regular polygon with $n$ sides. This completes the proof of Theorem 2.

An interesting application of the Schwarz-Christoffel correspondence is already apparent among cubic and quartic forms. Notice that equiangular polygons having three and four sides are respectively equilateral triangles and rectangles. Hence, if $F$ is a cubic form for which $F(x, 1)$ has three distinct real roots, then each of the sectors of the region $|F(x, y)| \leq 1$ has equal area, while if $F$ is a quartic form for which $F(x, 1)$ has four distinct real roots, then alternating sectors have equal areas.

Unfortunately, we have not been able to exploit further the Schwarz-Christoffel correspondence to prove that $M_{n}=D_{F_{n}^{*}}^{1 / n(n-1)} A_{F_{n}^{*}}$. This may be due in part to our inability to interpret the conformal property of the Schwarz-Christoffel maps in a meaningful way. If we consider the factor of the discriminant in the 


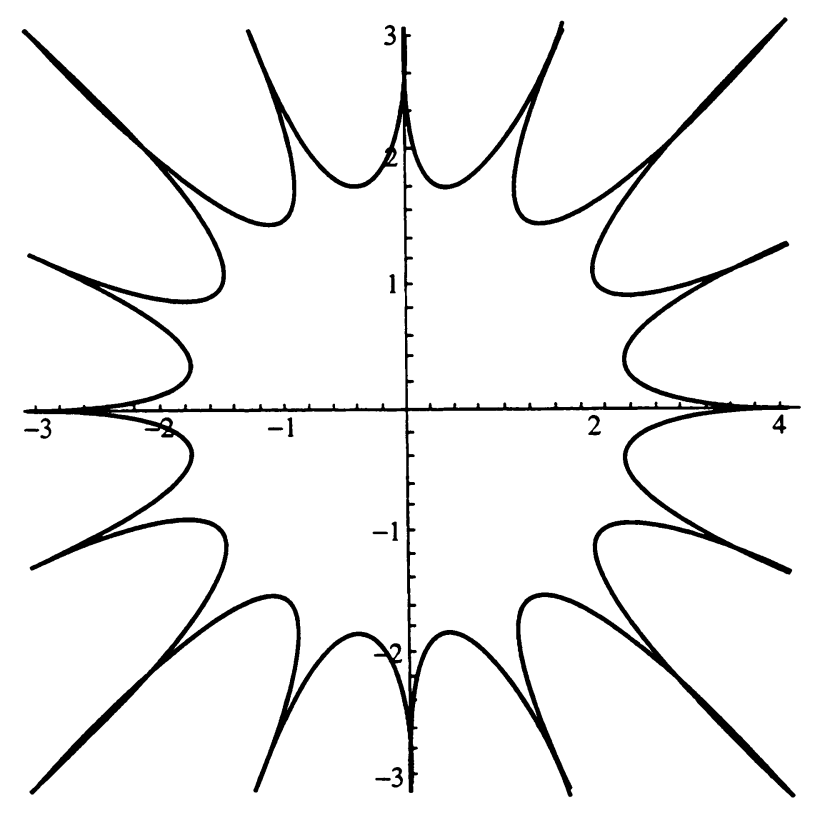

FigURE 6. $\left|F_{8}^{*}(x, y)\right|=1$

quantity $\left|D_{F}\right|^{1 / n(n-1)} A_{F}$ to be a normalizing factor, then the conjectures of $\S 1$ can be reformulated in the following natural way.

Conjecture 1. Among all forms $F$ of degree $n$ with a given discriminant and having a complete factorization over $\mathbb{R}$, the quantity $A_{F}$ is largest when the polygon corresponding to $F$ is a regular polygon.

Conjecture 2. If $\left\{F_{n}\right\}$ is a sequence of regular forms (i.e., forms for which $P\left(F_{n}\right)$ is an $n$-sided regular polygon) having a common discriminant $D$, then

$$
\lim _{n \rightarrow \infty} A_{F_{n}}=2 \pi \text {. }
$$

Note that this form of Conjecture 2 is equivalent to the earlier one since $\lim _{n \rightarrow \infty}|D|^{1 / n(n-1)}=1$ for any non-zero constant $D$. Note further that we can always normalize a non-degenerate form (i.e., arrange that $D_{F}= \pm 1$ ) by replacing $F$ with $F /\left|D_{F}\right|^{1 / 2(n-1)}$.

Now from the Schwarz-Christoffel correspondence, it is clear that the limiting value of the sequence $D_{F_{n}^{*}}^{1 / n(n-1)} A_{F_{n}^{*}}$ is the circumference of some circle. If it could be shown that the radius of this circle is an integer, then it would follow that the limiting value is $2 \pi$ since by Corollary 1 , each of the values $D_{F_{n}^{*}}^{1 / n(n-1)} A_{F_{n}^{*}}(n \geq 4)$ is less than 12. Computational evidence suggests that this is indeed the case (see Table 2). 
TABLE 2. Selected approximate numerical values of $D_{F_{n}^{*}}^{1 / n(n-1)} A_{F_{n}^{*}}$

\begin{tabular}{|r|l|l|}
\hline$n$ & $D_{F_{n}^{*}}^{1 / n-1)} A_{F_{n}^{*}}$ & $D_{F_{n}^{*}}^{1 / n(n-1)} A_{F_{n}^{*}} / 2 \pi$ \\
\hline 3 & 15.89974875 & 2.530523607 \\
4 & 11.77264037 & 1.873673908 \\
5 & 10.32275876 & 1.642918083 \\
10 & 8.273246977 & 1.316728152 \\
25 & 7.205128260 & 1.146731778 \\
50 & 6.809999892 & 1.083845145 \\
100 & 6.583462809 & 1.047790649 \\
250 & 6.424238441 & 1.022449303 \\
500 & 6.361968168 & 1.012538681 \\
1000 & 6.326792492 & 1.006940299 \\
& & \\
\hline
\end{tabular}

\section{ProOF OF TheOREM 4}

Put

$$
Q_{n}\left(u_{1}, \ldots, u_{n-3}\right)=\sum_{k=1}^{n} D_{F_{k}}^{1 / n(n-1)} \int_{0}^{1} \frac{d t}{F_{k}(1, t)^{2 / n}}
$$

where the $F_{k}$ are as in the statement of Theorem 3. In this section, we will demonstrate that the point $\left(u_{1}^{*}, \ldots, u_{n-3}^{*}\right)$ defined by

$$
u_{j}^{*}=\frac{1}{2}\left\{1-\frac{\tan \frac{\pi}{n}}{\tan (j+1) \frac{\pi}{n}}\right\}
$$

for $1 \leq j \leq n-3$, is a critical point of the function $Q_{n}$. The proof, which is rather technical in nature, appeals directly to the fact that the form $F_{n}^{*}$ belongs to this critical class and has a corresponding graph which is symmetric about each of its asymptotes. In particular, the proof makes use of the observation that $\left(u_{1}^{(k)}, \ldots, u_{n-3}^{(k)}\right)=\left(u_{1}^{*}, \ldots, u_{n-3}^{*}\right)$ for all $k$, at the point $\left(u_{1}^{*}, \ldots, u_{n-3}^{*}\right)$.

Put $G(X, Y)=X Y(X-Y)\left(X-w_{1} Y\right) \cdots\left(X-w_{n-3} Y\right)$ and let $M$ be defined by

$$
M\left(w_{1}, w_{2}, \ldots, w_{n-3}\right)=D_{G}^{1 / n(n-1)} \int_{0}^{1} \frac{d t}{G(1, t)^{2 / n}}
$$

for $0<w_{1}<w_{2}<\cdots<w_{n-3}<1$. Then

$$
Q_{n}\left(u_{1}, u_{2}, \ldots, u_{n-3}\right)=\sum_{k=1}^{n} M\left(u_{1}^{(k)}, u_{2}^{(k)}, \ldots, u_{n-3}^{(k)}\right)
$$


By the Chain Rule,

$$
\begin{aligned}
\frac{\partial}{\partial u_{l}} Q_{n}\left(u_{1}, u_{2}, \ldots, u_{n-3}\right) & =\sum_{k=1}^{n} \sum_{j=1}^{n-3} D_{j} M\left(u_{1}^{(k)}, \ldots, u_{n-3}^{(k)}\right) \frac{\partial u_{j}^{(k)}}{\partial u_{l}} \\
& =\sum_{j=1}^{n-3}\left\{\sum_{k=1}^{n} D_{j} M\left(u_{1}^{(k)}, \ldots, u_{n-3}^{(k)}\right) \frac{\partial u_{j}^{(k)}}{\partial u_{l}}\right\}
\end{aligned}
$$

for $l=1,2, \ldots, n-3$. Further, at the point $\left(u_{1}^{*}, \ldots, u_{n-3}^{*}\right)$ we have

$$
D_{j} M\left(u_{1}^{(k)}, \ldots, u_{n-3}^{(k)}\right)=D_{j} M\left(u_{1}^{*}, \ldots, u_{n-3}^{*}\right)
$$

which is independent of $k$. Hence, to show that

$$
\frac{\partial}{\partial u_{l}} Q_{n}\left(u_{1}^{*}, \ldots, u_{n-3}^{*}\right)=0
$$

it suffices to show that

for all $j$.

$$
\left.\sum_{k=1}^{n} \frac{\partial u_{j}^{(k)}}{\partial u_{l}}\right|_{\left(u_{i}^{*}, \ldots, u_{n-3}^{*}\right)}=0
$$

For simplicity, let us adopt the standard notation for cross ratios, namely

$$
\left[z_{1}, z_{2}, z_{3}, z_{4}\right]=\frac{\left(z_{4}-z_{3}\right)\left(z_{2}-z_{1}\right)}{\left(z_{4}-z_{2}\right)\left(z_{3}-z_{1}\right)} \text {. }
$$

With this notation, $u_{j}^{(k)}=\left[u_{n-k-1}, u_{n-k}, u_{n-k+1}, u_{n-k+1+j}\right]$, and so

$$
\sum_{k=1}^{n} u_{j}^{(k)}=\sum_{k=1}^{n} u_{j}^{(n-k+1)}=\sum_{k=1}^{n}\left[u_{k-2}, u_{k-1}, u_{k}, u_{k+j}\right] .
$$

Notice that $u_{l}$ appears in only four terms of the latter sum. Further,

$$
u_{j}^{*}=\left[u_{k-2}^{*}, u_{k-1}^{*}, u_{k}^{*}, u_{k+j}^{*}\right]
$$

since $u_{j}^{*}=u_{j}^{(k)}$ for all $k$. Hence, after some calculation, we find that

$$
\begin{aligned}
& \left.\sum_{k=1}^{n} \frac{\partial u_{j}^{(k)}}{\partial u_{l}}\right|_{\left(u_{i}^{*}, \ldots, u_{n-3}^{*}\right)} \\
& =\left.\frac{\partial}{\partial u_{l}} \sum_{k=1}^{n} u_{j}^{(k)}\right|_{\left(u_{l}^{*}, \ldots, u_{n-3}^{*}\right)} \\
& =u_{j}^{*}\left\{\frac{u_{l+1}^{*}-u_{l+2}^{*}}{\left(u_{l+1}^{*}-u_{l}^{*}\right)\left(u_{l+2}^{*}-u_{l}^{*}\right)}+\frac{u_{l-1}^{*}-u_{l-2}^{*}}{\left(u_{l-1}^{*}-u_{l}^{*}\right)\left(u_{l-2}^{*}-u_{l}^{*}\right)}\right\} \\
& \quad+u_{j}^{*}\left\{\frac{u_{l+j}^{*}-u_{l+j+1}^{*}}{\left(u_{l+j}^{*}-u_{l}^{*}\right)\left(u_{l+j+1}^{*}-u_{l}^{*}\right)}+\frac{u_{n-j+l}^{*}-u_{n-j+l-1}^{*}}{\left(u_{n-j+l}^{*}-u_{l}^{*}\right)\left(u_{n-j+l-1}^{*}-u_{l}^{*}\right)}\right\}
\end{aligned}
$$


We claim that

$$
\frac{u_{l+j}^{*}-u_{l+j+1}^{*}}{\left(u_{l+j}^{*}-u_{l}^{*}\right)\left(u_{l+j+1}^{*}-u_{l}^{*}\right)}+\frac{u_{n-j+l}^{*}-u_{n-j+l-1}^{*}}{\left(u_{n-j+l}^{*}-u_{l}^{*}\right)\left(u_{n-j+l-1}^{*}-u_{l}^{*}\right)}=0
$$

for all $l$ and $j$. This is equivalent to the statement that

$$
\left[u_{l-j-1}^{*}, u_{l-j}^{*}, u_{l}^{*}, u_{l+j}^{*}\right]=\left[u_{l+j+1}^{*}, u_{l+j}^{*}, u_{l}^{*}, u_{l-j}^{*}\right]
$$

for all $l$ and $j$. From the definition of $u_{j}^{*}$, we certainly have

$$
\begin{aligned}
& {\left[u_{l-j-1}^{*}, u_{l-j}^{*}, u_{l}^{*}, u_{l+j}^{*}\right]} \\
& \quad=\left[\tan (l-j) \frac{\pi}{n}, \tan (l-j+1) \frac{\pi}{n}, \tan (l+1) \frac{\pi}{n}, \tan (l+j+1) \frac{\pi}{n}\right]
\end{aligned}
$$

and

$$
\begin{aligned}
& {\left[u_{l+j+1}^{*}, u_{l+j}^{*}, u_{l}^{*}, u_{l-j}^{*}\right]} \\
& \quad=\left[\tan (l+j+2) \frac{\pi}{n}, \tan (l+j+1) \frac{\pi}{n}, \tan (l+1) \frac{\pi}{n}, \tan (l-j+1) \frac{\pi}{n}\right] .
\end{aligned}
$$

Further, since the curve $\left|F_{n}^{*}(x, y)\right|=1$ is symmetric about the line

$$
x \sin (l+1) \frac{\pi}{n}-y \cos (l+1) \frac{\pi}{n}=0
$$

and since cross ratios are invariant under $G L_{2}(\mathbb{R})$, it is apparent that

$$
\begin{aligned}
& {\left[\tan (l-j) \frac{\pi}{n}, \tan (l-j+1) \frac{\pi}{n}, \tan (l+1) \frac{\pi}{n}, \tan (l+j+1) \frac{\pi}{n}\right]} \\
& \quad=\left[\tan (l+j+2) \frac{\pi}{n}, \tan (l+j+1) \frac{\pi}{n}, \tan (l+1) \frac{\pi}{n}, \tan (l-j+1) \frac{\pi}{n}\right] .
\end{aligned}
$$

Hence

$$
\left[u_{l-j-1}^{*}, u_{l-j}^{*}, u_{l}^{*}, u_{l+j}^{*}\right]=\left[u_{l+j+1}^{*}, u_{l+j}^{*}, u_{l}^{*}, u_{l-j}^{*}\right]
$$

for all $l$ and $j$ as claimed.

It now follows that

for all $j$, and so

$$
\left.\sum_{k=1}^{n} \frac{\partial u_{j}^{(k)}}{\partial u_{l}}\right|_{\left(u_{i}^{*}, \ldots, u_{n-3}^{*}\right)}=0
$$

$$
\frac{\partial}{\partial u_{l}} Q_{n}\left(u_{1}^{*}, \ldots, u_{n-3}^{*}\right)=0
$$

for $l=1,2, \ldots, n-3$. This completes the proof of Theorem 4 .

\section{REFERENCES}

1. M. Abramowitz and I. Stegun, Handbook of mathematical functions, Dover, New York, 1965.

2. M.A. Bean, An isoperimetric inequality for the area of plane regions defined by binary forms, Compositio Math. 92 (1994), 115-131.

3. (1994), 204-207.

4. M.A. Bean and J.L. Thunder, Isoperimetric inequalities for volumes associated with decomposable forms. J. London Math. Soc. (to appear).

5. E. Bombieri and W.M. Schmidt. On Thue's equation, Invent. Math. 88 (1987), 69-81. 
6. B.W. Char et al., Maple library reference manual, Springer-Verlag, New York, 1991.

7. R.V. Churchill and J.W. Brown, Complex variables and applications, 5th ed., McGraw-Hill, New York, 1990.

8. E.T. Copson, An introduction to the theory of functions of a complex variable, Clarendon, Oxford, 1935.

9. L.E. Dickson, Algebraic invariants, Wiley, New York, 1914.

10. C. Hooley, On binary cubic forms, J. Reine Angew. Math. 226 (1967), 30-87.

11. K. Mahler, Zur Approximation algebraischer Zahlen III, Acta Math. 62 (1934), 91-166.

12. J. Mueller and W.M. Schmidt, Thue's equation and a conjecture of Siegel, Acta Math. 160 (1988), 207-247.

13. On the Newton polygon, Monatsh. Math. 113 (1992), 33-50.

14. G.C. Salmon, Modern higher algebra, 3rd ed., Dublin 1876, 4th ed., Dublin 1885 (reprinted New York, 1924).

15. W.M. Schmidt, Diophantine approximations and Diophantine equations, Lecture Notes in Math., vol. 1467, Springer-Verlag, New York, 1991.

16. L.J. Slater, Generalized hypergeometric functions, Cambridge, 1966.

17. A. Thue, Über Annäherungswerte algebraischer Zahlen, J. Reine Angew. Math. 135 (1909), 284-305.

18. E.T. Whittaker and G.N. Watson, Modern analysis, 4th ed., Cambridge 1927.

Department of Mathematics, University of Michigan, Ann Arbor, Michigan 48109

E-mail address: mbean@math.Isa.umich.edu 